\title{
The efficacy of postoperative radiotherapy for locally advanced rectal cancer without neoadjuvant therapy
}

\author{
Dehao Yu\#, Yu Wang", Yongxi Song, Peng Gao, Jingxu Sun, Xiaowan Chen, Yaoyuan Hu, Zhenning Wang \\ Department of Surgical Oncology and General Surgery, The First Hospital of China Medical University, Shenyang 110001, China \\ Contributions: (I) Conception and design: Z Wang, D Yu; (II) Administrative support: P Gao, Y Hu; (III) Provision of study materials or patients: \\ Y Wang, X Chen; (IV) Collection and assembly of data: J Sun, Y Song; (V) Data analysis and interpretation: P Gao; (VI) Manuscript writing: All \\ authors; (VII) Final approval of manuscript: All authors. \\ \#These authors contributed equally to this work. \\ Correspondence to: Zhenning Wang. Department of Surgical Oncology and General Surgery, The First Hospital of China Medical University, 155 \\ North Nanjing Street, Heping District, Shenyang 110001, China. Email: josieon826@sina.cn.
}

Background: The 2018 guidelines for treatment of cancer by site recommended by the National Comprehensive Cancer Network (NCCN) does not include a specific treatment recommendation for rectal cancer patients who did not receive preoperative chemoradiotherapy. The study objective was to evaluate whether postoperative radiotherapy was effective for such patients.

Methods: Survival was evaluated in 9,585 patients who underwent surgical resection for stage II or III rectal cancer and were registered in the Surveillance, Epidemiology, and End Results-Medicare database between 1992 and 2009. Overall survival (OS) benefit in propensity score (PS)-matched patient cohorts were estimated by Cox proportional hazards models.

Results: After stratification by chemotherapy regimen, the difference in OS of stage III patients between the no-treatment and radiotherapy groups was significant $(\mathrm{P}<0.001)$. However, there was no significant difference in OS of stage III patients between 5-fluorouracil (5-FU) and 5-FU/radiotherapy groups $(\mathrm{P}=0.267)$. Also, the difference in OS between the FOLFOX and FOLFOX/radiotherapy groups was not significant $(\mathrm{P}=0.649)$. The PS-matching analysis confirmed aforementioned results.

Conclusions: The evidence obtained in this study does not support postoperative radiotherapy for stage II rectal cancer or stage III patients who received postoperative chemotherapy. The use of postoperative radiotherapy for stage III patients who did not receive postoperative chemotherapy could increase OS.

Keywords: Rectal neoplasm; Surveillance, Epidemiology, and End Results (SEER) program; radiotherapy; chemotherapy

Submitted Feb 05, 2018. Accepted for publication Jun 15, 2018.

doi: $10.21037 /$ tcr.2018.06.19

View this article at: http://dx.doi.org/10.21037/tcr.2018.06.19

\section{Introduction}

Rectal cancer, which includes lesions located within $12 \mathrm{~cm}$ of the anal verge (1), is the most frequently diagnosed cancer and the most common cause of cancer-related death worldwide (2). Neoadjuvant chemoradiotherapy combined with total mesorectal excision is the recommended treatment for locally advanced (tumor- node-metastasis stage II or III) rectal cancer according to the 2018 Guidelines for Treatment of Cancer by Site recommended by the National Comprehensive Cancer Network (NCCN) (3). However, only an estimated 55\% of rectal cancer patients in the USA actually receive preoperative chemoradiotherapy $(4,5)$. The large minority of patients who do not receive neoadjuvant therapy as recommended by the NCCN, leads to the question of how 
to best treat these patients. The $2015 \mathrm{NCCN}$ treatment recommendations include adjuvant chemoradiotherapy for pathological stage II or III rectal cancer without preoperative treatment (6), but the 2018 update does not include a specific recommendation for patients who did not receive preoperative chemoradiotherapy (3), as it remains uncertain whether postoperative adjuvant radiotherapy is effective. Peng et al. found that postoperative radiotherapy had a significant survival benefit for patients with T3N0 rectal cancer, as compared with surgery alone (7). However, a prospective study by Kim et al. found no beneficial effect of postoperative radiotherapy on survival of patients with stage IIA rectal cancer after total mesorectal excision (8). Moreover, postoperative radiotherapy for locally advanced stage III rectal cancer has been associated with frequent, late-onset complications that were difficult to resolve and seriously impaired patient quality of life (9).

The aim of this study was to evaluate the effect of postoperative radiotherapy on survival of patients with locally advanced rectal cancer who did not receive preoperative chemoradiotherapy.

\section{Methods}

\section{Data source}

The cohort of this retrospective study was limited to patients aged $\geq 66$ years who underwent resection of rectal cancer between 1992 and 2009. Patient data were collected from the Surveillance, Epidemiology, and End Results (SEER)-Medicare database. The SEER database, which covers approximately $28 \%$ of the US population, includes patient demographics, tumor and disease characteristics, survival rates, and causes of death of individuals diagnosed with cancer (10). Medicare is the primary health insurer for approximately $97 \%$ of the US population aged $\geq 65$ years (11). The SEER-Medicare database is fully described elsewhere (12). The study protocol was approved by the Institutional Review Board of the First Hospital of China Medical University (Shenyang, China) and the use of the SEER-Medicare database was approved by the National Cancer Institute (Rockville, MD, USA).

\section{Patient selection}

The study cohort was limited to patients diagnosed with incident malignant primary rectum cancer (SEER cancer site codes 19.9 and 20.9) and who underwent primary tumor resection within 180 days of diagnosis. Patients who received any preoperative chemotherapy or radiotherapy, died within 30 days of resection, had stage I or IV tumors, a prior diagnosis of non-rectal cancer, incomplete pathological stage or diagnostic data, subscribed to a Medicare-sponsored health maintenance organization, or were not enrolled in Medicare parts A and B from 12 months preceding diagnosis to 60 months after diagnosis were excluded from analysis.

\section{Variables}

Five datasets in SEER-Medicare database (PEDSF, MEDPAR, Outpatient, $\mathrm{NCH}$, and DME) were used for the extraction of data. Status of radiotherapy, Age at diagnosis, year of diagnosis, gender, race, histological type, histological grade, number of lymph nodes examined, residence location, marital status, median household income, education level, pT category, and $\mathrm{pN}$ category were extracted from PEDSF. Intestinal obstruction was extracted from information of diagnosis in MEDPAR, Outpatient, NCH, and DME. To control for the effects of comorbidities, the analysis was adjusted by the Centers for Medicare and Medicaid Services Hierarchical Condition Category (HCC) using Medicare outpatient and inpatient claims for miscellaneous comorbidities within 12 months of cancer diagnosis. The HCC risk score summarized healthcare problems and forecasts future healthcare costs of a study population, as compared with the average Medicare beneficiary (13). Postoperative pathological stage was determined according to the criteria of the Union for International Cancer Control (UICC) tumor-node-metastasis staging system (14). Status and time of surgery were extracted from MEDPAR and NCH. Status and time of chemotherapy were extracted from MEDPAR, Outpatient, DME and NCH. The code determined chemotherapy was based on our previous study (15). Data on postoperative chemotherapy were obtained from claims filed within 9 months of surgery. The 5 -fluorouracil (5-FU) regimen included the use of 5-FU or capecitabine. The FOLFOX regimen included the use of oxaliplatin within 30 days of the first chemotherapy (5-FU) dose. Patients without any record of receiving chemotherapy within 1 year after surgery were included in the "no-treatment" group.

\section{Statistical analysis}

The chi-square test was used to compare differences in 
Table 1 Primary effects of variables included in the PS-matched models

\begin{tabular}{lll}
\hline Variables related to PS-matched models & Stage II patients & Stage III patients \\
\hline $\begin{array}{l}\text { Variables that significantly related to the patients' } \\
\text { probability of receiving radiotherapy among the } \\
\text { no-treatment and radiotherapy groups. }\end{array}$ & $\begin{array}{l}\text { Age at diagnosis, level of } \\
\text { education, year at diagnosis, } \\
\text { race, HCC risk score, median } \\
\text { household income, histologic } \\
\text { type, pT category }\end{array}$ & $\begin{array}{l}\text { Intestinal obstruction, age at diagnosis, year } \\
\text { at diagnosis, HCC risk score, marital status, } \\
\text { histologic type, pN category, gender }\end{array}$ \\
$\begin{array}{l}\text { Variables that significantly related to the patients' } \\
\text { probability of receiving radiotherapy among the } \\
\text { 5-FU and 5-FU/radiotherapy groups. }\end{array}$ & $\begin{array}{ll}\text { Intestinal obstruction, residence } \\
\text { location, pT category }\end{array}$ & $\begin{array}{l}\text { Intestinal obstruction, age at diagnosis, year at } \\
\text { diagnosis, residence location, marital status, } \\
\text { gender }\end{array}$ \\
\hline
\end{tabular}

PS, propensity score; HCC, Hierarchical Condition Categories; 5-FU, 5-fluorouracil.

demographic and tumor characteristics among groups. For univariate analysis of overall survival (OS), KaplanMeier estimates of cumulative survival were compared by the log-rank test. Because the choice of radiotherapy was likely to have been confounded by several patient variables, propensity score (PS)-matched analysis was performed to adjust for differences in risk when estimating the effects of treatment on survival $(16,17)$. PS matching reduces bias by assembling a sample in which confounding factors are balanced between the no-radiotherapy and radiotherapy groups. Univariate logistic regression was used to identify factors related to treatment selection $(\mathrm{P}<0.05)$. Multivariate logistic regression was used to estimate the PS of each group (Table 1). Matching by PS was performed using the psmatch2 module available with the STATA 14.0 software package (Stata LLC, College Station, TX, USA). A Cox proportional hazards model was used for adjusted analysis of variables that were identified as significantly associated with survival by univariate analysis. Hazard ratios (HRs) with $95 \%$ confidence intervals (CIs) were calculated. Statistical analyses were performed and graphics were created using SAS 9.4 software (SAS Institute, Cary, NC, USA), STATA 14.0, and IBM SPSS Statistics for Windows, version 20.0. (IBM Corp., Armonk, NY, USA). A probability P value $<0.05$ was considered statistically significant.

\section{Results}

\section{Patient characteristics}

The demographics of the 9,585 selected patients (stage II, $\mathrm{n}=4,587$; stage III, $\mathrm{n}=4,998$ ) are shown in Table 2. Compared with non-radiotherapy, radiotherapy was more common in male $(\mathrm{P}<0.001)$, younger individuals $(\mathrm{P}<0.001)$, year at diagnosis before $2000(\mathrm{P}<0.001)$, white race $(\mathrm{P}=0.016)$, married $(\mathrm{P}<0.001)$, poor histological grade $(\mathrm{P}<0.001)$,
T4b category $(\mathrm{P}<0.001), \mathrm{N} 1-\mathrm{N} 2$ category $(\mathrm{P}<0.001)$, nonintestinal obstruction $(\mathrm{P}<0.001)$, number of examined lymph nodes $<12(\mathrm{P}=0.035), 5$-FU chemotherapy $(\mathrm{P}<0.001)$.

\section{Stage II patients}

The results of univariate and multivariate analyses of stage II patients by Cox proportional hazards regression are shown in Table 3. Univariate analysis found that radiotherapy $(\mathrm{HR}=0.591,95 \% \mathrm{CI}: 0.524-0.666 ; \mathrm{P}<0.001$ ) was associated with survival. In multivariate analysis, radiotherapy was not significantly associated with survival (HR =0.940; 95\% CI: 0.811-1.090; $\mathrm{P}=0.415$ ).

There was no significant difference in OS with or without radiotherapy among stage II patients $(\mathrm{P}=0.073$, Figure $1 A)$. Also, there was no significant difference in OS between the 5-FU and 5-FU/radiotherapy treatment groups $(\mathrm{P}=0.914$, Figure $1 B)$. In the PS-matched cohorts, the differences in OS in the No-treatment and Radiotherapy groups were not significant $(\mathrm{P}=0.430$, Figure $1 C)$. The difference in OS between the 5-FU and 5-FU/radiotherapy groups was not significant $(\mathrm{P}=0.815$, Figure $1 D)$.

\section{Stage III patients}

The results of univariate and multivariate analyses and Cox proportional hazards regression for patients with stage III tumors are shown in Table 4. Univariate and multivariate analyses showed that radiotherapy was associated with OS ( $\mathrm{HR}=0.651$ and 0.870 ; $95 \%$ CI: $0.598-0.707$ and $0.791-$ 0.956; $\mathrm{P}<0.001$ and $\mathrm{P}=0.004$, respectively).

The effectiveness of radiotherapy was also evaluated separately in three treatment-group pairs, i.e., notreatment $v$ s. radiotherapy, 5-FU vs. 5-FU/radiotherapy, and FOLFOX vs. FOLFOX/radiotherapy (Figures 2). 
Table 2 Clinicopathological characteristics of the included rectal cancer patients

\begin{tabular}{|c|c|c|c|}
\hline Clinicopathologic characteristics & \multicolumn{2}{|c|}{ Number of patients (\%) } & $P$ value \\
\hline Gender & & & $<0.001$ \\
\hline Female & $3,547(73.8)$ & 1,258 (26.2) & \\
\hline Age at diagnosis (years) & & & $<0.001$ \\
\hline $72-75$ & $1,234(63.7)$ & $704(36.3)$ & \\
\hline $76-81$ & $1,897(71.4)$ & $759(28.6)$ & \\
\hline$>81$ & $2,292(88.1)$ & $310(11.9)$ & \\
\hline Residence location* & & & 0.015 \\
\hline Year at diagnosis & & & $<0.001$ \\
\hline 1992-1996 & $1,592(67.3)$ & $773(32.7)$ & \\
\hline $1997-2000$ & $1,287(66.0)$ & $662(34.0)$ & \\
\hline 2001-2004 & $2,103(72.1)$ & $813(27.9)$ & \\
\hline 2005-2009 & $1,815(77.1)$ & $540(22.9)$ & \\
\hline Race & & & 0.016 \\
\hline White & $5,801(70.4)$ & 2,442 (29.6) & \\
\hline Black & $454(75.7)$ & $146(24.3)$ & \\
\hline Divorced + widowed & $2,719(75.8)$ & $869(24.2)$ & \\
\hline Other & $216(77.1)$ & $64(22.9)$ & \\
\hline Level of education & & & 0.010 \\
\hline 1st quartile & $1,706(71.3)$ & $688(28.7)$ & \\
\hline 2nd quartile & $1,658(69.1)$ & $741(30.9)$ & \\
\hline 3rd quartile & $1,675(70.0)$ & $718(30.0)$ & \\
\hline 4th quartile & 1,758 (73.3) & $641(26.7)$ & \\
\hline Median household income & & & 0.131 \\
\hline 1st quartile & $1,697(70.9)$ & $696(29.1)$ & \\
\hline 2nd quartile & $1,685(70.4)$ & 708 (29.6) & \\
\hline 3rd quartile & $1,672(69.7)$ & $728(30.3)$ & \\
\hline 4th quartile & $1,743(72.7)$ & $656(27.3)$ & \\
\hline
\end{tabular}

Table 2 (continued) 
Table 2 (continued)

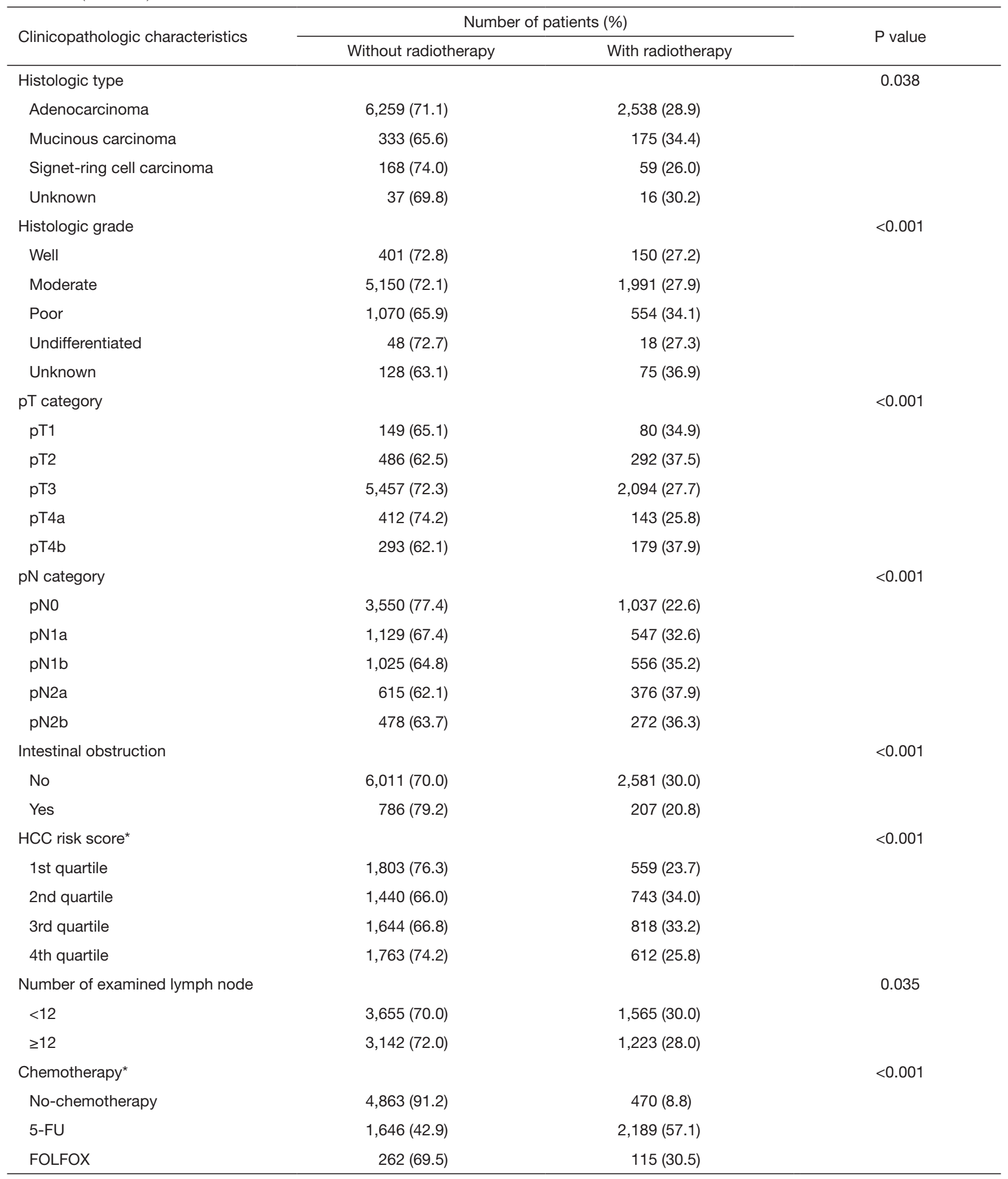

*, variables have missing data. HCC, Hierarchical Condition Categories; HR hazard ratio; 5-FU, 5-fluorouracil; Cl, confidence interval. 
Table 3 Univariate and multivariate analyses of OS of stage II patients

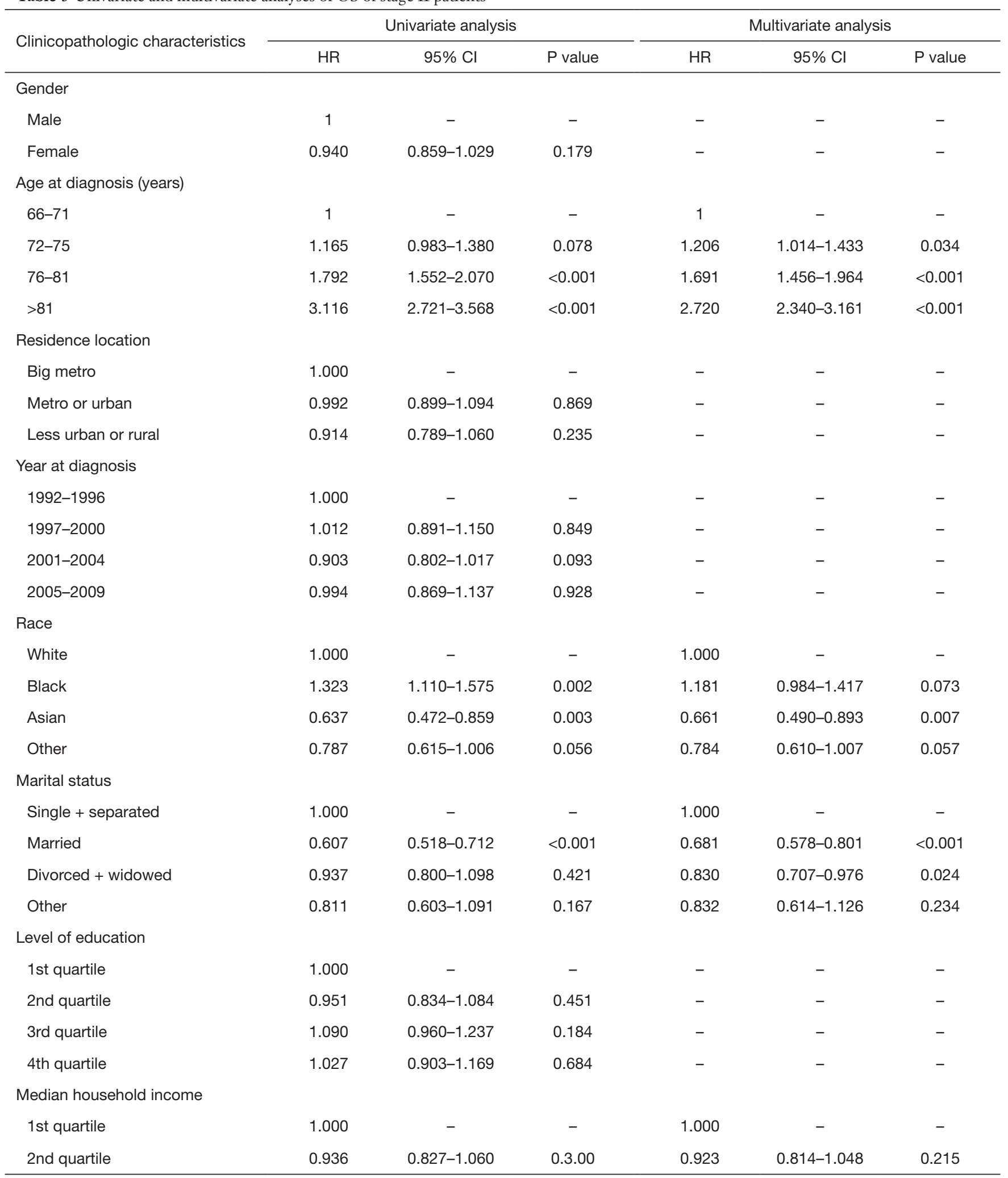

Table 3 (continued) 
Table 3 (continued)

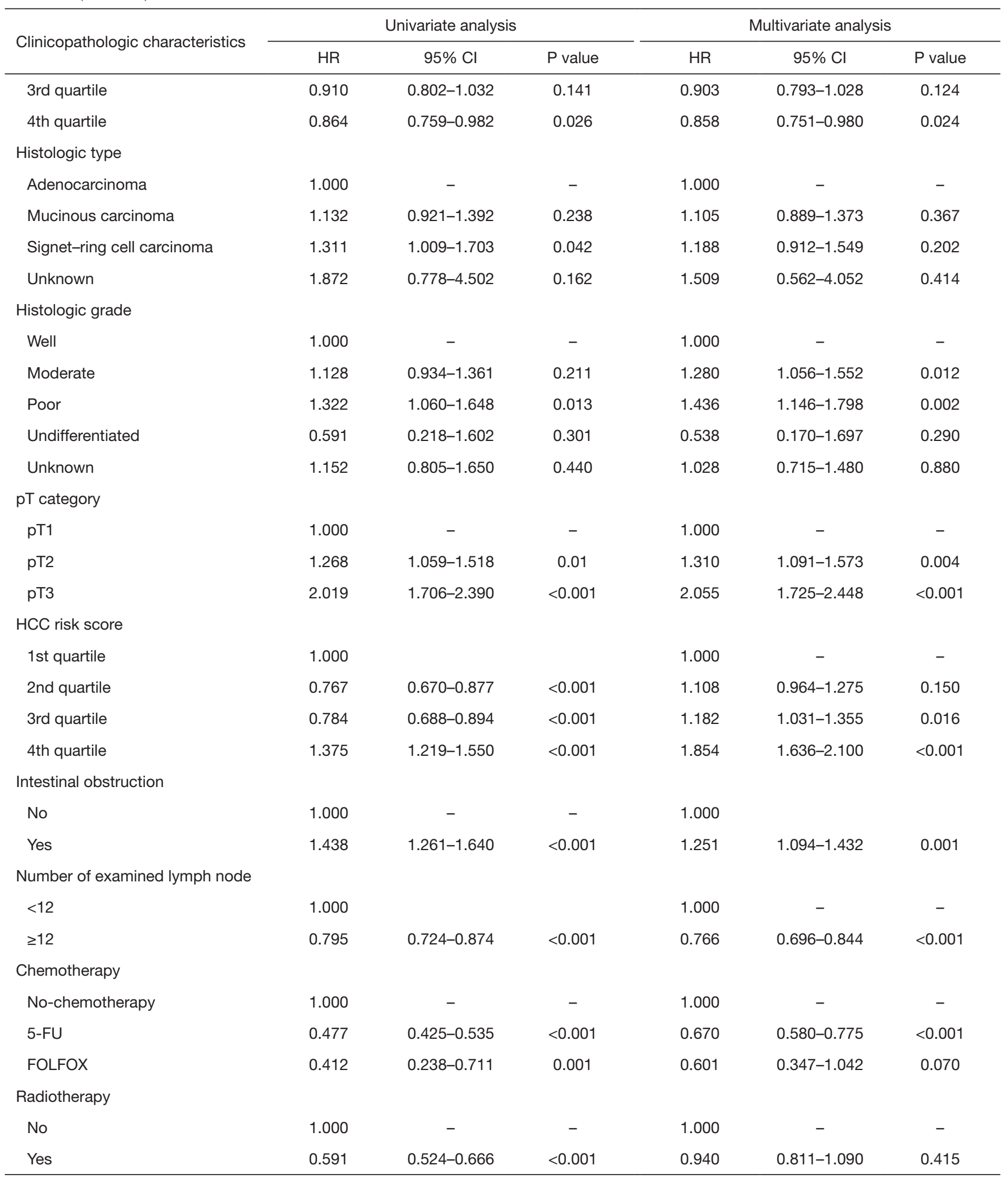

OS, overall survival; HCC, Hierarchical Condition Categories; HR hazard ratio; 5-FU, 5-fluorouracil; Cl, confidence interval. 
A

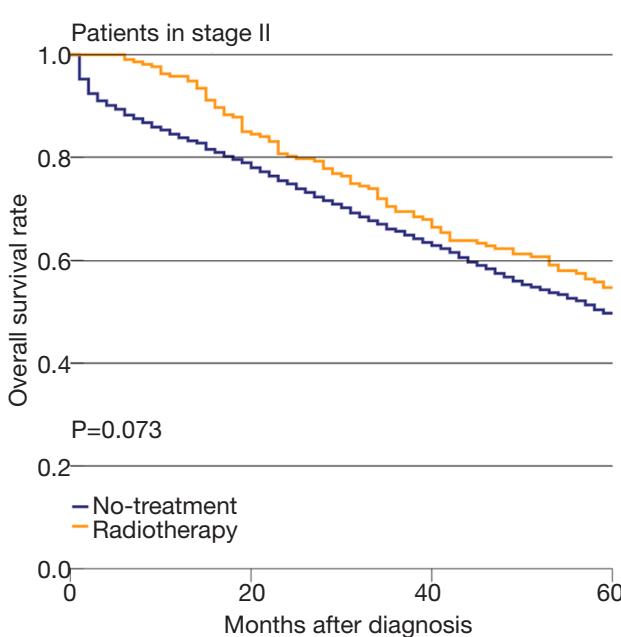

C

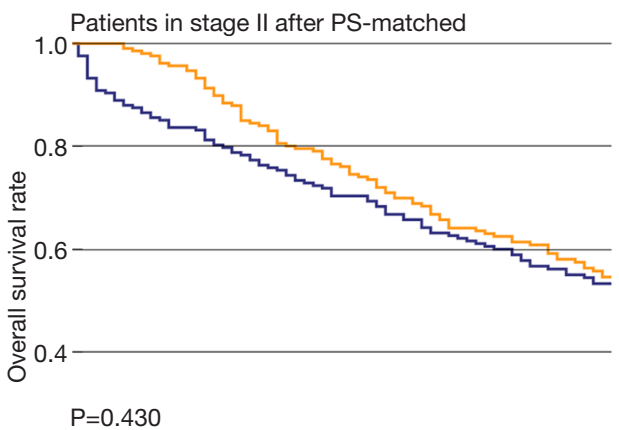

0.2

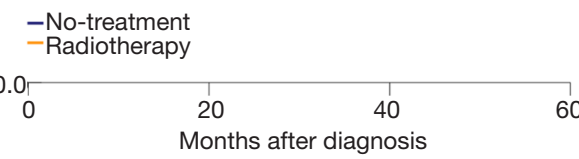

B

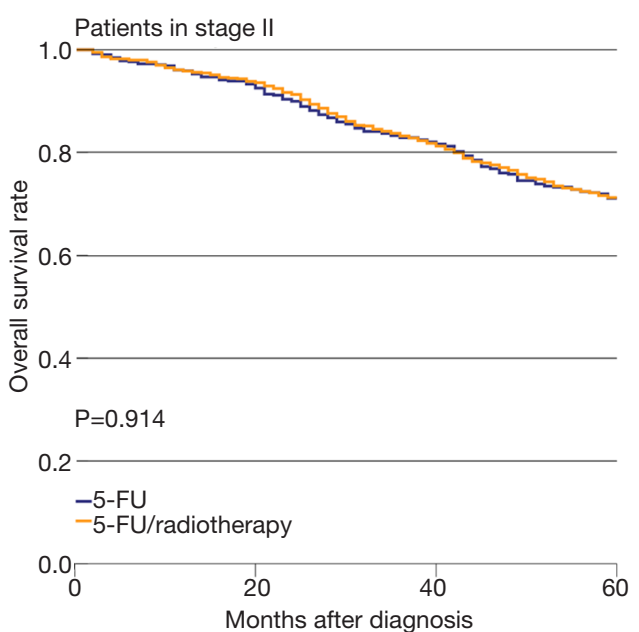

D Patients in stage II after PS-matched

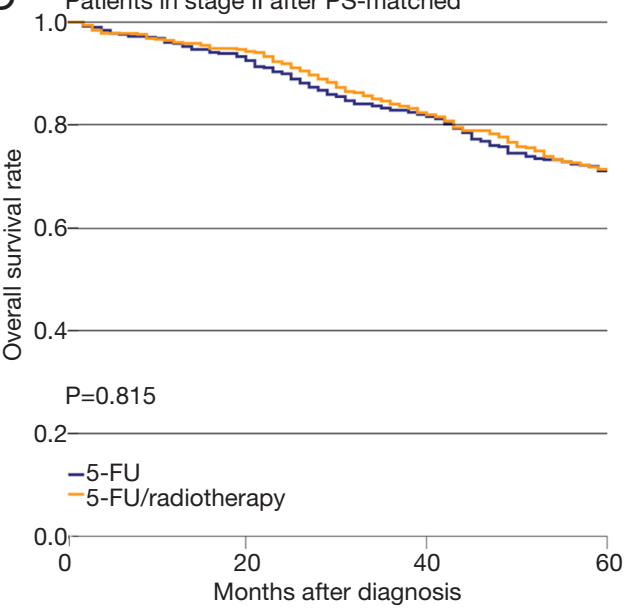

Figure 1 Kaplan-Meier estimates of OS in PS-matched and non-PS-matched patient cohorts with stage II disease who received different postoperative treatments. (A) OS rate of non-PS-matched patient cohorts with no-treatment or radiotherapy; (B) OS rate in PSmatched patient cohorts with no-treatment or radiotherapy; (C) OS rate of non-PS-matched patient cohorts treated with 5-FU or 5-FU/ radiotherapy; (D) OS rate in PS-matched patient cohorts treated with 5-FU or 5-FU/radiotherapy. OS, overall survival; PS, propensity score; 5-FU, 5-fluorouracil.

The difference in OS of stage III patients between the notreatment and radiotherapy groups was significant $(\mathrm{P}<0.001$, Figure $2 A$ ). However, the difference in OS between the $5-\mathrm{FU}$ and 5-FU/radiotherapy groups was not significant $(\mathrm{P}=0.267$, Figure $2 B)$. Also, the difference in OS between the FOLFOX and FOLFOX/radiotherapy groups was not significant $(\mathrm{P}=0.649$, Figure $2 C)$. PS matching did not change the significance of the differences in OS between the No-treatment and Radiotherapy groups $(\mathrm{P}<0.001$, Figure $3 A$ ). The difference in OS between the $5-\mathrm{FU}$ and 5-FU/radiotherapy groups remained insignificant after PS matching $(\mathrm{P}=0.174$, Figure $3 B)$. The sizes of the FOLFOX and FOLFOX/radiotherapy groups were too small to generate a PS-matched cohort.

\section{Discussion}

Surgery is performed to remove the bulk of the tumor, but may fail to achieve a circumference completely clear of tumor cells. Radiotherapy accomplishes just the opposite by clearing the superficial surface, but not the bulk of the tumor (18). Hence, the rationale for combining 
Table 4 Univariate and multivariate analyses of OS of stage III patients

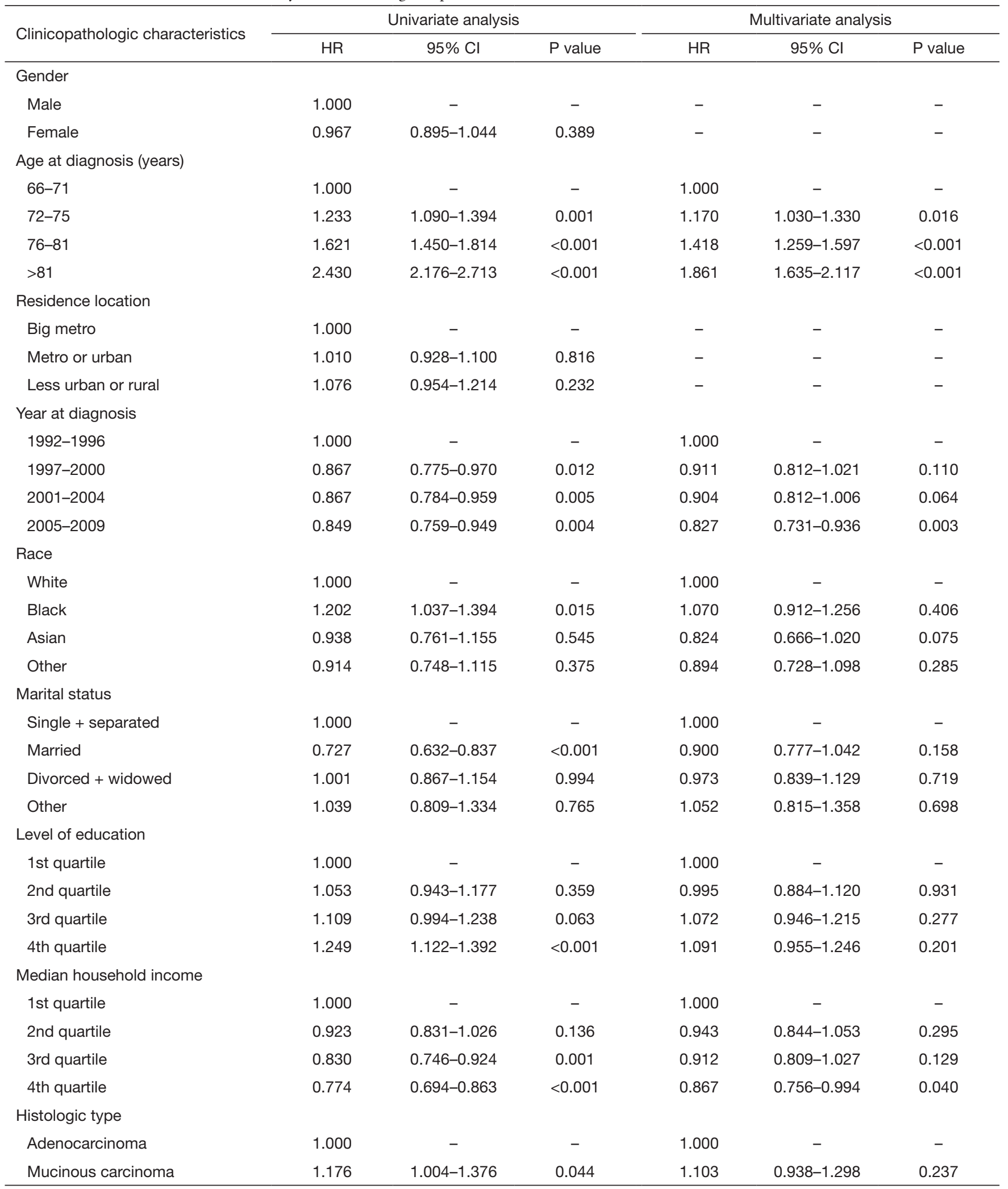

Table 4 (continued) 
Table 4 (continued)

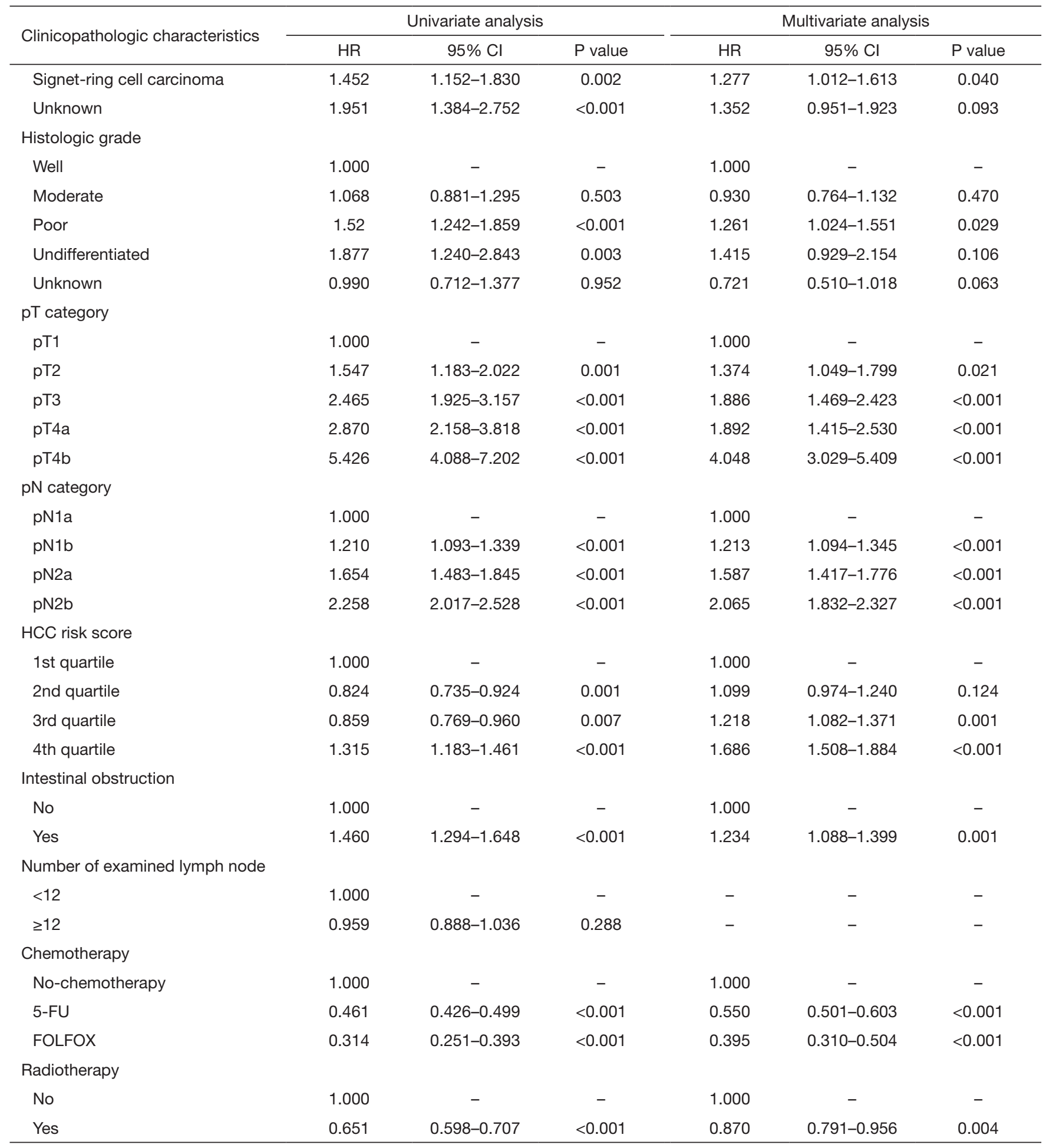

OS, overall survival; HCC, Hierarchical Condition Categories; HR hazard ratio; 5-FU, 5-fluorouracil; Cl, confidence interval. 

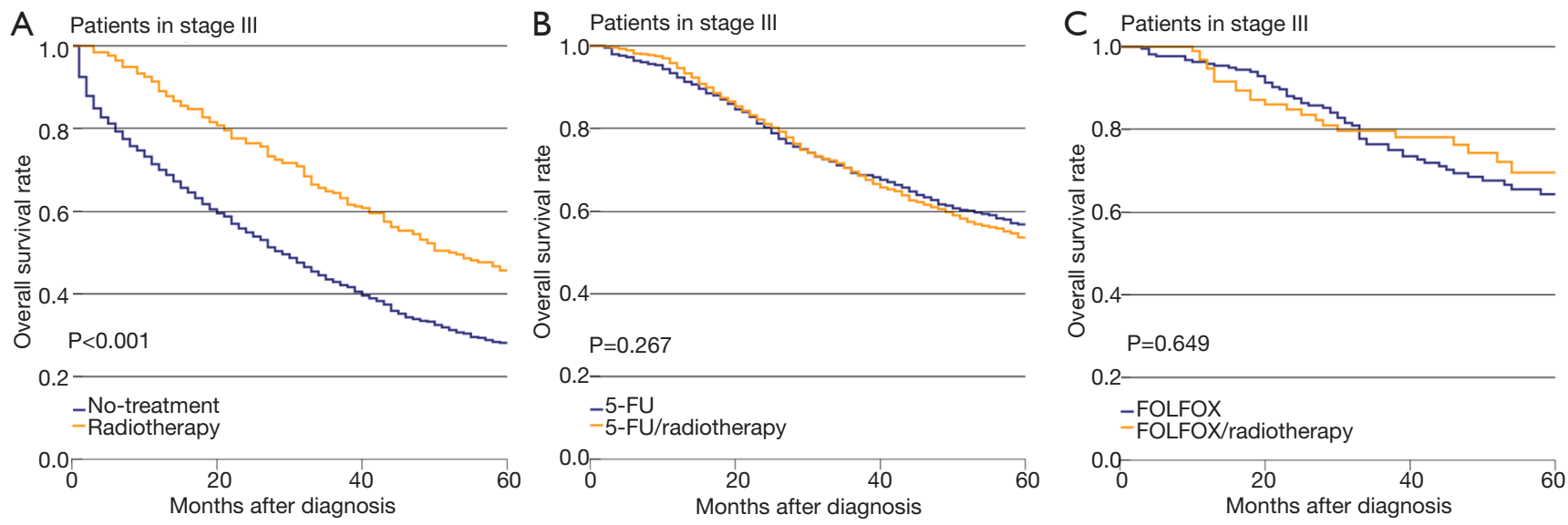

Figure 2 Kaplan-Meier estimates of OS in non-PS-matched patient cohorts with stage III disease who received different postoperative treatments. (A) OS rate of non-PS-matched patient cohorts with no-treatment or radiotherapy; (B) OS rate of non-PS-matched patient cohorts treated with 5-FU or 5-FU/radiotherapy; (C) OS rate of non-PS-matched patient cohorts treated with FOLFOX or FOLFOX/ radiotherapy. OS, overall survival; PS, propensity score; 5-FU, 5-fluorouracil.

A

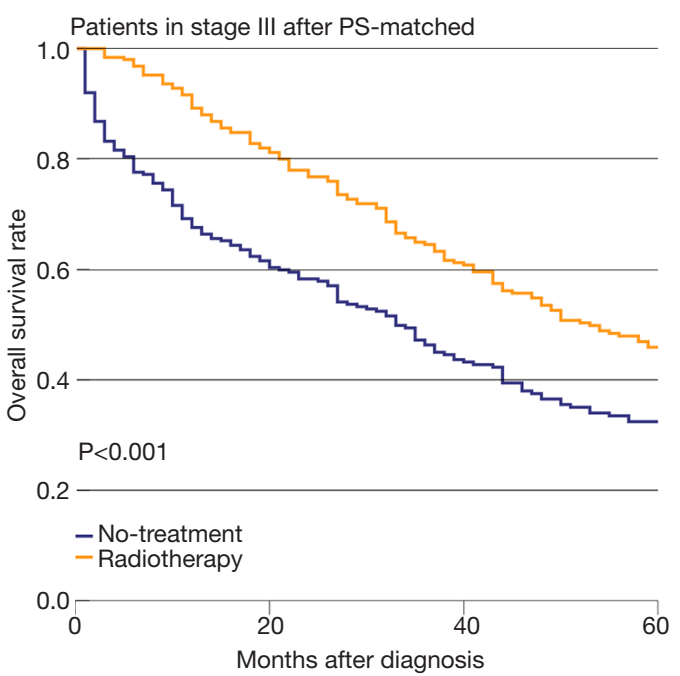

B

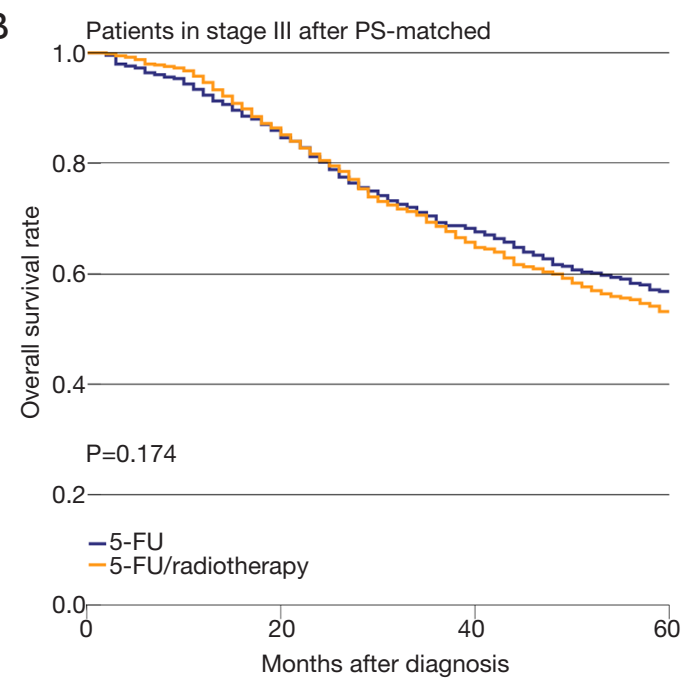

Figure 3 Kaplan-Meier estimates of OS in PS-matched patient cohorts with stage III disease and different postoperative treatment. (A) OS rate of patients with No-treatment or radiotherapy; (B) OS rate of patients treated with 5-FU or 5-FU/radiotherapy. OS, overall survival; PS, propensity score; 5-FU, 5-fluorouracil.

radiotherapy with surgery for treatment of primary and recurrent rectal cancer is to remove the tumor and kill micrometastases at the periphery. Previous studies suggested that preoperative radiotherapy was more effective than postoperative radiotherapy. The CAO/ARO/AIO94 trial, a large prospective, randomized trial conducted by the German Rectal Cancer Study Group, compared preoperative versus postoperative radiotherapy for clinical stage II/III rectal cancer (19) and found that preoperative therapy was associated with significant reductions in local recurrence and treatment-associated toxicity, which can be explained by better tumor oxygenation during preoperative, as compared with postoperative, treatment.

Not all patients received radiotherapy before surgery, while the evidence for postoperative radiotherapy combined with radical surgery for locally advanced rectal cancer is not strong. A 1997 phase III trial conducted by the European Organization for Research and Treatment of Cancer failed 
to demonstrate that postoperative radiotherapy improved OS or local control after resection of locally advanced rectal cancer (20). However, a recent analysis of SEER data on the effect of radiotherapy in 4,724 patients with T3N0 rectal cancer found that postoperative radiation was associated with a significant decrease in the risk of cancer-related death, as compared with surgery alone, while preoperative radiation was not (7). Some studies have suggested that postoperative radiotherapy might not be necessary in selected patients, such as those with stage II rectal cancer, which has better oncologic outcomes after surgery than advanced stage disease (21-23). If postoperative radiotherapy is overtreatment, the associated side-effects cannot be ignored. Komori et al. reported multiple complications, including gut inflammation and edema, after postoperative irradiation for locally advanced stage III rectal carcinoma. Late-onset complications were frequent, difficult to resolve, and seriously impaired patient quality of life (9).

The aim of the present study was to determine whether postoperative radiotherapy provided a survival benefit to patients with locally advanced rectal cancer who did not receive preoperative chemoradiotherapy. Because the curative effect of radiotherapy may differ with disease stage, stage II and stage III patients were evaluated separately. The addition of postoperative radiation was not associated with a significant improvement of OS among stage II patients. In stage III patients, postoperative radiotherapy did significantly improve OS, as compared with the no treatment group. However, there were no significant differences in OS in the patient groups receiving FOLFOX or 5-FU chemotherapy versus those who received no postoperative radiotherapy. Although chemotherapy was recommended for all stage III patients, some could not complete the chemotherapy course because of anaphylaxis or severe complications. According to the results of this study, these patients should receive postoperative radiotherapy.

After that, we found patients who received radiotherapy in stage II and stage III hold diverse benefits. A few reports demonstrated stage II and stage III rectal cancer patients who received radiotherapy gained different benefits of adjuvant therapy, especially the adjuvant radiotherapy. Recent studies showed both neoadjuvant radiotherapy and adjuvant radiotherapy improved survival outcomes of stage III rectal cancer patients while had little effect on stage II cases (23-25). Consequently, these differences indicated that stage influenced the survival outcomes of patients who were suffered from advanced rectal cancer, while more advanced cases, especially in stage III, experiencing a greater survival benefit after received radiotherapy (26).

Meanwhile, we find several reports demonstrated chemoradiotherapy prefer to have higher benefits in improving local control rather than OS. A recent study found that rectal cancer patients received radiotherapy had an improved local control, however, that was not significantly in improving OS (27). Additionally, patients with postoperative radiotherapy were observed to have reduced local recurrence rate in rectal cancer $(28,29)$. Due to the limitation of SEER-Medicare database that the information on the prevalence of recurrence during the follow-up period was not well supported, our analysis focused on patients' OS. In view of the results of the aforesaid studies, we believed that patients with rectal cancer could benefit from postoperative radiotherapy by reducing local recurrence. Even though we didn't find the benefit of postoperative radiotherapy on OS in stage III patients with postoperative chemotherapy and stage II patients, there was no reason to deny the effect of postoperative radiotherapy in improving the patients' local control.

Analysis of the SEER-Medicare data had some limitations. First, although both PS matching and Cox proportional hazards models were used to eliminate known confounders, the effect of patient selection could not be completely eliminated because of the retrospective design of this exploratory study. Hence, these findings should be verified in a prospective study. Second, although the SEER-Medicare database is a powerful resource to identify overall trends and interesting patterns in a large patient sample, it is limited by the scope of available information because the database lacks some data important for risk stratification, such as radiotherapy dose and fractionation, perineural and lymphovascular invasion, distance from the anal verge, circumferential margin status, carcinoembryonic antigen level. Patients with perineural and lymphovascular invasion were demonstrated to be a more aggressive phenotype, suggesting that they may be benefit from adjuvant therapy (30). A recent study reported that only recommending patients with tumors in the lower rectum to receive radiotherapy but omitting those with tumors in the mid and upper rectum was inadvisable (31). Meanwhile, a study demonstrated that the effect of radiotherapy became stronger as the distance from the anal verge increased (32). Therefore, upper and mid rectal cancer may also have better postoperative receiving radiotherapy. Also, the involvement of positive circumferential resection margin was reported to influence the effect of radiotherapy on rectal cancer (33). 
Certainly, it is important to add these unavailable variables in the PS matching and Cox proportional hazards models in future research.

\section{Conclusions}

The evidence obtained in this study does not support postoperative radiotherapy for stage II rectal cancer or stage III patients who received postoperative chemotherapy. The use of postoperative radiotherapy for stage III patients who did not receive postoperative chemotherapy could increase OS.

\section{Acknowledgments}

Funding: This work was supported by The Doctoral Scientific Research Startup Foundation of Liaoning Province (201601138), Clinical Capability Construction Project for Liaoning Provincial Hospitals (LNCCC-A01-2014), National Key R\&D Program of China (MOST2016YFC1303200, MOST-2016YFC1303202).

\section{Footnote}

Conflicts of Interest: All authors have completed the ICMJE uniform disclosure form (available at http://dx.doi. org/10.21037/tcr.2018.06.19). The authors have no conflicts of interest to declare.

Ethical Statement: The authors are accountable for all aspects of the work in ensuring that questions related to the accuracy or integrity of any part of the work are appropriately investigated and resolved. The study was conducted in accordance with the Declaration of Helsinki (as revised in 2013). The study protocol was approved by the Institutional Review Board of the First Hospital of China Medical University (Shenyang, China) and the use of the SEER-Medicare database was approved by the National Cancer Institute (Rockville, MD, USA). Prior informed consent was not required because SEER-Medicare registry data were de-identified.

Open Access Statement: This is an Open Access article distributed in accordance with the Creative Commons Attribution-NonCommercial-NoDerivs 4.0 International License (CC BY-NC-ND 4.0), which permits the noncommercial replication and distribution of the article with the strict proviso that no changes or edits are made and the original work is properly cited (including links to both the formal publication through the relevant DOI and the license). See: https://creativecommons.org/licenses/by-nc-nd/4.0/.

\section{References}

1. Nelson H, Petrelli N, Carlin A, et al. Guidelines 2000 for colon and rectal cancer surgery. J Natl Cancer Inst 2001;93:583-96.

2. Siegel RL, Miller KD, Jemal A. Cancer statistics, 2016. CA Cancer J Clin 2016;66:7-30.

3. Rectal cancer V.1. NCCN Clinical Practical Guidelines in Oncology. 2018.

4. Barton MK. Delivery of neoadjuvant chemoradiation for patients with stage II and III rectal cancer is suboptimal. CA Cancer J Clin 2016;66:357-8.

5. Sineshaw HM, Jemal A, Thomas CR Jr, et al. Changes in treatment patterns for patients with locally advanced rectal cancer in the United States over the past decade: An analysis from the National Cancer Data Base. Cancer 2016;122:1996-2003.

6. Rectal cancer V.1. NCCN Clinical Practical Guidelines in Oncology. 2015.

7. Peng LC, Milsom J, Garrett K, et al. Surveillance, epidemiology, and end results-based analysis of the impact of preoperative or postoperative radiotherapy on survival outcomes for T3N0 rectal cancer. Cancer Epidemiol 2014;38:73-8.

8. Kim JS, Kim NK, Min BS, et al. Adjuvant radiotherapy following total mesorectal excision for stage IIA rectal cancer: is it beneficial? Int J Colorectal Dis 2010;25:1103-10.

9. Komori K, Kimura K, Kinoshita T, et al. Complications associated with postoperative adjuvant radiation therapy for advanced rectal cancer. Int Surg 2014;99:100-5.

10. National Cancer Institute: Surveillance, Epidemiology and End Results (SEER). Available online: http:// seercancergov/about/overviewhtml

11. Potosky AL, Riley GF, Lubitz JD, et al. Potential for cancer related health services research using a linked Medicare-tumor registry database. Med Care 1993;31:732-48.

12. SEER-Medicare: About the data files. Available online: http://appliedresearchcancergov/seermedicare/aboutdata/

13. Ash AS, Ellis RP, Pope GC, et al. Using diagnoses to describe populations and predict costs. Health Care Financ Rev 2000;21:7-28.

14. Sobin LH, Gospodarowicz MK, Wittekind C. TNM classification of malignant tumours, 7th edition. Oxford: 
Wiley-Blackwell, 2009.

15. Gao P, Song YX, Sun JX, et al. Which is the best postoperative chemotherapy regimen in patients with rectal cancer after neoadjuvant therapy? BMC Cancer 2014;14:888.

16. Rubin DB. Estimating causal effects from large data sets using propensity scores. Ann Intern Med 1997;127:757-63.

17. Leuven E, Sianesi B. PSMATCH2: Stata module to perform full mahalanobis and propensity score matching, common support graphing, and covariate imbalance testing. Statistical Software Components 2005.

18. Glimelius B. Pre- or postoperative radiotherapy in rectal cancer--more to learn? Radiother Oncol 2001;61:1-5.

19. Sauer R, Becker H, Hohenberger W, et al. Preoperative versus postoperative chemoradiotherapy for rectal cancer. N Engl J Med 2004;351:1731-40.

20. Arnaud JP, Nordlinger B, Bosset JF, et al. Radical surgery and postoperative radiotherapy as combined treatment in rectal cancer. Final results of a phase III study of the European Organization for Research and Treatment of Cancer. Br J Surg 1997;84:352-7.

21. Lai LL, Fuller CD, Kachnic LA, et al. Can pelvic radiotherapy be omitted in select patients with rectal cancer? Semin Oncol 2006;33:S70-4.

22. Fujita S, Yamamoto S, Akasu T, et al. Outcome of patients with clinical stage II or III rectal cancer treated without adjuvant radiotherapy. Int J Colorectal Dis 2008;23:1073-9.

23. Law WL, Ho JW, Chan R, et al. Outcome of anterior resection for stage II rectal cancer without radiation: the role of adjuvant chemotherapy. Dis Colon Rectum 2005;48:218-26.

24. Li Q, Li Y, Dai W, et al. Adjuvant radiotherapy improves cause specific survival in stage II, not stage III mucinous carcinoma of the rectum. BMC Cancer 2017;17:80.

Cite this article as: Yu D, Wang Y, Song Y, Gao P, Sun J, Chen $\mathrm{X}, \mathrm{Hu} \mathrm{Y}$, Wang Z. The efficacy of postoperative radiotherapy for locally advanced rectal cancer without neoadjuvant therapy. Transl Cancer Res 2018;7(4):922-935. doi: 10.21037/ tcr.2018.06.19
25. Yang L, Ma Q, Yu YY, et al. Efficacy of surgery and adjuvant therapy in older patients with colorectal cancer: a STROBE-compliant article. Medicine (Baltimore) 2014;93:e266.

26. Moody JS, Sawrie SM, Kozak KR, et al. Stage-specific survival differences associated with postoperative radiotherapy for gastrointestinal cancers. J Gastrointest Cancer 2008;39:86-99.

27. Huh JW, Kim HC, Park HC, et al. Is Chemoradiotherapy Beneficial for Stage IV Rectal Cancer? Oncology 2015;89:14-22.

28. Bouvet M, Milas M, Giacco GG, et al. Predictors of recurrence after local excision and postoperative chemoradiation therapy of adenocarcinoma of the rectum. Ann Surg Oncol 1999;6:26-32.

29. Borg M, Sutherland P, Stapleton A, et al. Outcome of postprostatectomy radiotherapy in one institution. Australas Radiol 2006;50:475-80.

30. Cienfuegos JA, Rotellar F, Baixauli J, et al. Impact of perineural and lymphovascular invasion on oncological outcomes in rectal cancer treated with neoadjuvant chemoradiotherapy and surgery. Ann Surg Oncol 2015;22:916-23.

31. Braendengen M, Tveit KM, Berglund A, et al. Randomized phase III study comparing preoperative radiotherapy with chemoradiotherapy in nonresectable rectal cancer. J Clin Oncol 2008;26:3687-94.

32. Holm T, Johansson H, Rutqvist LE, et al. Tumour location and the effects of preoperative radiotherapy in the treatment of rectal cancer. Br J Surg 2001;88:839-43.

33. Marijnen CA, Nagtegaal ID, Kapiteijn E, et al. Radiotherapy does not compensate for positive resection margins in rectal cancer patients: report of a multicenter randomized trial. Int J Radiat Oncol Biol Phys 2003;55:1311-20. 